\title{
On Two Conjectures of Abel Grassmann's Groupoids
}

\author{
Xiaohong Zhang ${ }^{1, * \mathbb{D}}$, Yingcang $\mathrm{Ma}^{2}$ and Peng $\mathrm{Yu}^{1}$ \\ 1 School of Arts and Sciences, Shaanxi University of Science \& Technology, Xi'an 710021, China; \\ yupeng@sust.edu.cn \\ 2 School of Science, Xi'an Polytechnic University, Xi'an 710048, China; mayingcang@126.com \\ * Correspondence: zhangxiaohong@sust.edu.cn
}

Received: 13 May 2019; Accepted: 16 June 2019; Published: 20 June 2019

\begin{abstract}
The quasi-cancellativity of Abel Grassmann's groupoids (AG-groupoids) are discussed and two conjectures are partially solved. First, the following conjecture is proved to be true: every AG-3-band is quasi-cancellative. Moreover, a new notion of AG-(4,1)-band is proposed, and it is also proved that every AG-(4,1)-band is quasi-cancellative. Second, the notions of left (right) quasi-cancellative AG-groupoids and power-cancellative AG-groupoids are proposed, and the following results are obtained: for an $\mathrm{AG}^{*}$-groupoid or $\mathrm{AG}^{* *}$-groupoid, it is left quasi-cancellative if and only if it is right quasi-cancellative; for a power-cancellative and locally power-associative AG-groupoid, it is left quasi-cancellative if and only if it is right quasi-cancellative. Finally, a general result is proved, that for any AG-groupoid, if it is left quasi-cancellative then it is right quasi-cancellative.
\end{abstract}

Keywords: Abel-Grassmann's groupoid (AG-groupoid); AG-3-band; AG*-groupoid; quasicancellative; power-cancellative

\section{Introduction}

Group and semigroup are two kinds of important algebraic structures, which are mathematical abstractions of symmetry in real world. Their algebraic operations satisfy the law of association. At the same time, some algebraic structures that do not satisfy associative laws (collectively called non-associative algebra systems) also have many research results, such as Lie algebras, Jordan algebras, non-associative rings and so on. Abel-Grassmann's groupoid (studied in this paper) is also a kind of non-associative algebraic system.

The concept of Abel-Grassmann's groupoid (AG-groupoid) was proposed by Kazim and Naseerudd in 1972 [1], and it is also called left almost semigroup (LA-semigroup). Until now, as a kind of non-associative algebraic structure, AG-groupoid is still an active research direction [2-17]. AG-groupoid has important applications in flock theory and geometry (see the introduction in Reference [9]). In this paper, we focus on quasi-cancellativity of AG-groupoids.

In Reference [9], the authors introduced the notion of quasi-cancellative AG-groupoid: an AG-groupoid $S$ is quasi-cancellative if for any $x, y \in S$,

(i) $\quad x=x y$ and $y^{2}=y x$ imply that $x=y$,

(ii) $\quad x=y x$ and $y^{2}=x y$ imply that $x=y$.

The authors discussed some basic properties of quasi-cancellative AG-groupoids in Reference [9]. Moreover, the following two conjectures were pointed out:

Conjecture 1. Conditions (i) and (ii) above are equivalent for AG-groupoids.

Conjecture 2. Every AG-3-band is quasi-cancellative. 
In this paper, we will prove that Conjective 2 above is true. Moreover, we will propose a new notion of AG-(4,1)-band, and prove that every AG-(4,1)-band is quasi-cancellative. By introducing some new concepts of left quasi-cancellative, right quasi-cancellative AG-groupoids and power- cancellative AG-groupoids, we will discuss the relationships among some special kinds of AG-groupoids. Moreover, we will give some interesting examples.

\section{Preliminaries}

Definition 1. ([1-3]) Assume that $(S, *)$ is a groupoid. $(S, *)$ is called an Abel-Grassmann's groupoid (AG-groupoid) or a left almost semigroup (LA-semigroup), if it satisfies left invertive law, that is,

$$
\forall a, b, c \in S,(a * b) * c=(c * b) * a \text {. }
$$

Proposition 1. ([1]) Let $(S, *)$ be an AG-groupoid. Then the medial law holds, that is,

$$
\forall a, b, c, d \in S,(a * b) *(c * d)=(a * c) *(b * d) .
$$

Definition 2. ([4-6]) Let $(S, *)$ be an AG-groupoid. If all elements of $S$ are idempotents, then $S$ is called an AG-band.

It is easy to see that in an AG-band $S$, for any $a, b, c \in S,(a * b) * a=a *(b * a)$ and $(a * b) * c=(a * c) *(b * c)$, $(a * b) * b=b * a$.

Definition 3. ([5]) Let $(S, *)$ be an AG-groupoid, $a \in S$ arbitrary element. If $(a * a) * a=a *(a * a)=a$, we say that $a$ is a 3-potent. AG-groupoid $(S, *)$ is a 3-band (or AG-3-band) if all of its elements are 3-potents.

Proposition 2. ([5]) Let $(S, *)$ be an AG-groupoid. If $a \in S$ is a 3-potent, then $a^{2}$ is idempotent.

Definition 4. ([8]) Let $(S, *)$ be an AG-groupoid, a $a$ S an arbitrary element. If all products of a of length 4 are equal to $a^{2}$, we say that $a$ is a 4-potent. The AG-groupoid $S$ is an AG-4-band (or a 4-band) if all of its elements are 4-potents.

Definition 5. ([5]) An AG-groupoid $S$ is called an $A G^{*}$-groupoid if it satisfies one of the following equivalent weak associative laws:

$$
\begin{aligned}
& \forall a, b, c \in S,(a * b) * c=b *(a * c) . \\
& \forall a, b, c \in S,(a * b) * c=b *(c * a) .
\end{aligned}
$$

It is easy to see that in an $\mathrm{AG}^{*}$-groupoid $S$, for any $a, b, c \in S, b *(a * c)=b *(c * a)$.

Definition 6. ([5,13]) An AG-groupoid $S$ is called $A G^{* *-g r o u p o i d ~ i f ~ i t ~ s a t i s f i e s ~}$

$$
\forall a, b, c \in S, a *(b * c)=b *(a * c) \text {. }
$$

Definition 7. ([9]) Let $(S, *)$ be an AG-groupoid. $S$ is called a quasi-cancellative AG-groupoid, if for any $a, b \in S$,

(i) $a=a * b$ and $b^{2}=b * a$ imply $a=b$;

(ii) $a=b * a$ and $b^{2}=a * b$ imply $a=b$.

Proposition 3. ([9]) Every AG-band is quasi-cancellative. 


\section{Quasi-Cancellativity of AG-3-Bands and AG- $(4,1)$-Bands}

In this section, we prove two new properties of AG-groupoids, by these new results we prove that the Conjective 2 in [9] is true. Moreover, we introduce a new notion of AG-(4,1)-band, and discuss the relationships between AG-(4,1)-bands and quasi-cancellative AG-groupoids.

Lemma 1. Let $(S, *)$ be an AG-groupoid, $x, y \in S$ arbitrary two elements. If $x=x * y$ and $y^{2}=y * x$, then

(1) $x^{2}=x^{2} * y^{2}=x * x^{2}$;

(2) $y^{2} * y^{2}=y^{2} * x^{2}=\left(x^{2} * x\right) * y$;

(3) $y^{2} * y^{2}=y^{2} * x=x$;

(4) $x^{2} * y=y^{2} * x$.

Proof. (1) Since $x=x * y$ and $y^{2}=y * x$, applying Definition 1 and Proposition 1 we have

$$
\begin{gathered}
x^{2}=(x * y) *(x * y)=(x * x) *(y * y)=x^{2} * y^{2} ; \\
x^{2} * y^{2}=(x * x) *(y * x)=(x * y) *(x * x)=x * x^{2} .
\end{gathered}
$$

(2) By Definition 1 and Proposition 1,

$$
\begin{gathered}
y^{2} * y^{2}=(y * x) *(y * x)=(y * y) *(x * x)=y^{2} * x^{2} ; \\
y^{2} * x^{2}=(y * x) * x^{2}=\left(x^{2} * x\right) * y .
\end{gathered}
$$

(3) By Definition 1 and Proposition 1,

$$
\begin{gathered}
y^{2} * y^{2}=(y * x) * y^{2}=(y * x) *(y * y)=y^{2} *(x * y)=y^{2} * x ; \\
y^{2} * x=(y * y) * x=(x * y) * y=x * y=x .
\end{gathered}
$$

(4) From Definition 1 we have

$$
x^{2} * y=(x * x) * y=(y * x) * x=y^{2} * x .
$$

Lemma 2. Suppose that $(S, *)$ is an AG-groupoid, $x, y \in S$ are arbitrary two elements. If $x=y * x$ and $y^{2}=x * y$, then

(1) $x^{2}=y^{2} * x^{2}=x^{2} * x$;

(2) $y^{2} * y^{2}=x^{2} * y^{2}=\left(y^{2} * y\right) * x^{2}$;

(3) $y^{2} * x^{2}=x^{2} * y^{2}=x^{2}$;

(4) $x * y^{2}=y^{2} * y^{2}, x^{2} * y=x^{2}$.

Proof. (1) Since $x=y * x$ and $y^{2}=x * y$, applying Definition 1 and Proposition 1 we have

$$
\begin{gathered}
x^{2}=(y * x) *(y * x)=(y * y) *(x * x)=y^{2} * x^{2} ; \\
y^{2} * x^{2}=(x * y) * x^{2}=(x * y) *(x * x)=x^{2} *(y * x)=x^{2} * x .
\end{gathered}
$$

(2) By (1), Definition 1 and Proposition 1, we can get that

$$
\begin{gathered}
y^{2} * y^{2}=(x * y) *(x * y)=(x * x) *(y * y)=x^{2} * y^{2} \\
x^{2} * y^{2}=\left(x^{2} * x\right) * y^{2}=\left(x^{2} * x\right) *(y * y)=\left(x^{2} * y\right) *(x * y)=\left(x^{2} * y\right) * y^{2}=\left(y^{2} * y\right) * x^{2} .
\end{gathered}
$$


(3) Applying (1), (2) and Proposition 1, we have

$$
\begin{gathered}
y^{2} * x^{2}=(y * y) *(x * x)=(y * x) *(y * x)=x * x=x^{2} ; \\
x^{2} * y^{2}=\left(y^{2} * y\right) * x^{2}=\left(y^{2} * y\right) *\left(x^{2} * x\right)=\left(y^{2} * x^{2}\right) *(y * x)=\left(y^{2} * x^{2}\right) * x=x^{2} * x=x^{2} .
\end{gathered}
$$

(4) Using Proposition 1 and Definition 1, we get that

$$
\begin{gathered}
x * y^{2}=(y * x) * y^{2}=(y * x) *(y * y)=(y * y) *(x * y)=y^{2} * y^{2} ; \\
x^{2} * y=(x * x) * y=(y * x) * x=x * x=x^{2} .
\end{gathered}
$$

Theorem 1. Assume $\left(S,{ }^{*}\right)$ is an AG-3-band, then $\left(S,{ }^{*}\right)$ is quasi-cancellative.

Proof. Suppose that $(S, *)$ is an AG-3-band, then $(a * a) * a=a *(a * a)=a$ for any $a \in S$.

(1) First, we prove that $\left(x=x * y, y^{2}=y * x\right) \Rightarrow x=y$.

By Definition 3, we have

$$
x * x^{2}=x, y^{2} * y=y, y * y^{2}=y .
$$

Appying Lemma 1 (1), $x^{2}=x * x^{2}=x$. Using Lemma 1 (2) and (3) we have

$$
x=y^{2} * y^{2}=(y * y) * y^{2}=\left(y^{2} * y\right)=y * y=y^{2} .
$$

Hence,

$$
x=y^{2}=y * x=y * y^{2}=y .
$$

(2) Second, we prove that $\left(x=y * x, y^{2}=x * y\right) \Rightarrow x=y$.

By Definition 3, we have

$$
x^{2} * x=x, y^{2} * y=y .
$$

Appying Lemma $2(1), x^{2}=x^{2} * x=x$. From this, using Lemma 2 (4) we get that

$$
x=x^{2}=x^{2} * y=x * y=y^{2} .
$$

Hence,

$$
x=y^{2}=x * y=y^{2} * y=y .
$$

Definition 8. An AG-groupoid $S$ is called an AG-(4,1)-band if it satisfies: $\forall a \in S, a^{2} * a^{2}=a$.

Note that, by Definition 4 and Definition 8, the notions of AG-4-band and AG-(4,1)-band are different. The follwong examples show that an AG-4-band may be not an AG-(4,1)-band and an AG-(4,1)-band may be not an AG-4-band.

Example 1. Denote $S=\{1,2,3,4,5,6,7\}$, define operations $*$ on $S$ as Table 1 . We can verify that $(S, *)$ is an $A G-4-$ band, but it is not an $A G-(4,1)$-band, since

$$
7^{2} * 7^{2}=2 \neq 7
$$


Table 1. The operation * on AG-4-band $S$.

\begin{tabular}{llllllll}
\hline$*$ & $\mathbf{1}$ & $\mathbf{2}$ & $\mathbf{3}$ & $\mathbf{4}$ & $\mathbf{5}$ & $\mathbf{6}$ & $\mathbf{7}$ \\
\hline $\mathbf{1}$ & 1 & 2 & 2 & 5 & 6 & 4 & 7 \\
$\mathbf{2}$ & 2 & 2 & 2 & 5 & 6 & 4 & 2 \\
$\mathbf{3}$ & 2 & 2 & 3 & 5 & 6 & 4 & 2 \\
$\mathbf{4}$ & 6 & 6 & 6 & 4 & 2 & 5 & 6 \\
$\mathbf{5}$ & 4 & 4 & 4 & 6 & 5 & 2 & 4 \\
$\mathbf{6}$ & 5 & 5 & 5 & 2 & 4 & 6 & 5 \\
$\mathbf{7}$ & 7 & 2 & 2 & 5 & 6 & 4 & 2 \\
\hline
\end{tabular}

Example 2. Let $S=\{1,2,3,4,5\}$, define operations $*$ on $S$ as Table 2 . We can verify that $(S, *)$ is an $A G-(4,1)-b a n d$, but it is not an AG-4-band, since

$$
((4 * 4) * 4) * 4=4 \neq 5=4 * 4 \text {. }
$$

Table 2. The operation * on AG-(4,1)-band $S$.

\begin{tabular}{llllll}
\hline$*$ & $\mathbf{1}$ & $\mathbf{2}$ & $\mathbf{3}$ & $\mathbf{4}$ & $\mathbf{5}$ \\
\hline $\mathbf{1}$ & 1 & 1 & 1 & 1 & 1 \\
$\mathbf{2}$ & 1 & 2 & 2 & 4 & 5 \\
$\mathbf{3}$ & 1 & 2 & 3 & 4 & 5 \\
$\mathbf{4}$ & 1 & 4 & 4 & 5 & 2 \\
$\mathbf{5}$ & 1 & 5 & 5 & 2 & 4 \\
\hline
\end{tabular}

Moreover, we can verify that $(S, *)$ is not an AG-3-band and AG-band.

By, Definition 4 and Definition 8 , we can easy to prove the following proposition (the proof is omitted).

Proposition 4. Let $(S, *)$ be an AG-groupoid. Then $S$ is both an AG-4-band and an AG-(4,1)-band if and only if $S$ is an AG-band.

Theorem 2. Every AG-(4,1)-band is quasi-cancellative.

Proof. Let $(S, *)$ be an AG-(4,1)-band, then $a^{2} * a^{2}=a$ for any $a \in S$.

(1) First, we prove that $\left(x=x * y, y^{2}=y * x\right) \Rightarrow x=y$.

By Definition 8, we have $y^{2} * y^{2}=y$. Using Lemma 1 (3), we get that $x=y^{2} * y^{2}=y$.

(2) Second, we prove that $\left(x=y * x, y^{2}=x * y\right) \Rightarrow x=y$.

Applying Definition 8, $y^{2} * y^{2}=y$. From this, using Lemma 2 (2), (3) and (4) we have

$$
x^{2}=x^{2} * y=x^{2} * y^{2}=y^{2} * y^{2}=y .
$$

From this, using Lemma 2 (1),

$$
x^{2}=x^{2} * x=y * x=x
$$

Hence, $x=x^{2}=y$.

Note that, for the AG-4-band $S$ in Example 1, since

$$
\begin{aligned}
& 2=2 * 7,7^{2}=2=7 * 2, \text { but } 7 \neq 2 ; \\
& 2=7 * 2,7^{2}=2=2 * 7, \text { but } 2 \neq 7 .
\end{aligned}
$$


This means that the AG-4-band $S$ in Example 1 is not quasi-cancellative, that is, an AG-4-band may not be a quasi-cancellative AG-groupoid. The following example shows that a quasi-cancellative AG-4-band may be not an AG-band.

Example 3. Denote $S=\{1,2,3,4,5,6,7\}$, define operations $*$ on $S$ as Table 3. We can verify that $(S, *)$ is a quasi-cancellative AG-4-band, but it is not an AG-band, since

$$
7 * 7=3 \neq 7 .
$$

Table 3. The operation * on quasi-cancellative AG-4-band $S$.

\begin{tabular}{llllllll}
\hline $\boldsymbol{*}$ & $\mathbf{1}$ & $\mathbf{2}$ & $\mathbf{3}$ & $\mathbf{4}$ & $\mathbf{5}$ & $\mathbf{6}$ & $\mathbf{7}$ \\
\hline $\mathbf{1}$ & 1 & 2 & 2 & 5 & 6 & 4 & 2 \\
$\mathbf{2}$ & 2 & 2 & 2 & 5 & 6 & 4 & 2 \\
$\mathbf{3}$ & 2 & 2 & 3 & 5 & 6 & 4 & 7 \\
$\mathbf{4}$ & 6 & 6 & 6 & 4 & 2 & 5 & 6 \\
$\mathbf{5}$ & 4 & 4 & 4 & 6 & 5 & 2 & 4 \\
$\mathbf{6}$ & 5 & 5 & 5 & 2 & 4 & 6 & 5 \\
$\mathbf{7}$ & 2 & 2 & 7 & 5 & 6 & 4 & 3 \\
\hline
\end{tabular}

\section{Left (Right) Quasi-Cancellative and Power-Cancellative AG-Groupoids}

In this section, we introduce the new concepts of left quasi-cancellative, right quasi-cancellative and power-cancellative AG-groupoids, and discuss the relationships among them. Moreover, we give some interesting examples.

Definition 9. Suppose that $(S, *)$ is an AG-groupoid. $S$ is called a left quasi-cancellative AG-groupoid, if for any $a, b \in S$,

(i) $a=a * b$ and $b^{2}=b * a$ imply $a=b$; $S$ is called a right quasi-cancellative AG-groupoid, if for any $a, b \in S$,

(ii) $a=b * a$ and $b^{2}=a * b$ imply $a=b$.

Theorem 3. Assume that $(S, *)$ is an $A G^{*}$-groupoid. Then $S$ is left quasi-cancellative if and only if it is right quasi-cancellative.

Proof. Suppose that $(S, *)$ is an $\mathrm{AG}^{*}$-groupoid, then $(a * b) * c=b *(a * c)=b *(c * a)$ for any $a, b, c \in S$.

(1) Assume that $S$ is left quasi-cancellative, $x=y * x$ and $y^{2}=x * y, x, y \in S$.

Applying Lemma 2 (1), $x^{2}=x^{2} * x$. And, by Definition $5, x^{2}=x^{2} * x=(x * x) * x=x *(x * x)=x * x^{2}$. Then

$$
x^{2}=x^{2} * x \text { and } x^{2}=x * x^{2} .
$$

Since $S$ is left quasi-cancellative, so, from above we get that $x^{2}=x$. From this, using Lemma 2 (4) we have

$$
x=x^{2}=x^{2} * y=x * y .
$$

Hence, $x=y * x=x * y$. Therefore,

$$
x=x * y \text { and } y^{2}=x * y=y * x .
$$

Applying the left quasi-cancellative law, we get that $x=y$. This means that $S$ is right quasi-cancellative.

(2) Assume that $S$ is right quasi-cancellative, $x=x * y$ and $y^{2}=y * x, x, y \in S$.

By Lemma 1 (1), $x^{2}=x * x^{2}$. And, from this and Definition $5, x^{2} * x=(x * x) * x=x *(x * x)=x * x^{2}=x^{2}$. Then

$$
x^{2}=x * x^{2} \text { and } x^{2}=x^{2} * x .
$$


Using right quasi-cancellative, from above we get that $x^{2}=x$. Moreover, from $y^{2}=y * x$, by Definition 5 we have

$$
y^{2} * x=(y * x) * x=x *(y * x)=x * y^{2} .
$$

From this, applying Lemma 1 (3) we get that

$$
y^{2} * y^{2}=y^{2} * x=x * y^{2}
$$

That is,

$$
x=y^{2} * x(\text { Lemma } 1(3)) \text {, and } y^{2} * y^{2}=x * y^{2} .
$$

It follows that $x=y^{2}$ from the right quasi-cancellative law. Hence,

$$
x=y^{2}=y * x \text { and } y^{2}=x=x * y .
$$

From this and the right quasi-cancellative law, we have $x=y$. This means that $S$ is left quasi- cancellative.

Theorem 4. Assume that $(S, *)$ is an $A G^{* *}$-groupoid. Then $S$ is left quasi-cancellative if and only if it is right quasi-cancellative.

Proof. Let $(S, *)$ be an AG**-groupoid, then $a *(b * c)=b *(a * c)$ for any $a, b, c \in S$.

(1) Assume that $S$ is left quasi-cancellative, $x=y * x$ and $y^{2}=x * y, x, y \in S$.

By Lemma 2 (1) and (2), we have

$$
x^{2}=y^{2} * x^{2} \text { and } y^{2} * y^{2}=x^{2} * y^{2} .
$$

Applying left quasi-cancellative, we get $x^{2}=y^{2}$. Moreover, using Lemma 2 (1) and (4) we can get that

$$
x * x^{2}=(y * x) * x^{2}=\left(x^{2} * x\right) * y=x^{2} * y=x^{2} .
$$

It follows that

$$
x^{2}=x^{2} * x \text { and } x^{2}=x * x^{2} .
$$

From this, by left quasi-cancellative, we have $x=x^{2}$. Hence, $x * y=y^{2}=x^{2}=x=y * x$ and

$$
x=x * y \text { and } y^{2}=y * x .
$$

Using the left quasi-cancellative law again, we get that $x=y$. It follows that $S$ is right quasi-cancellative.

(2) Assume that $S$ is right quasi-cancellative, $x=x * y$ and $y^{2}=y * x, x, y \in S$.

By Definition 6, Lemma 1 (3), (2), (1) and (4) we get that

$$
\begin{gathered}
x^{2} * x^{2}=x^{2} *(x * x)=x *\left(x^{2} * x\right)=\left(y^{2} * x\right) *\left(x^{2} * x\right)=\left(y^{2} * x^{2}\right) * x^{2}=\left(y^{2} * y^{2}\right) * x^{2}=x * x^{2}=x^{2} . \\
x^{2} * x=x^{2} *(x * y)=x *\left(x^{2} * y\right)=x *\left(y^{2} * x\right)=y^{2} *(x * x)=y^{2} * x^{2}=x .
\end{gathered}
$$

It follows that

$$
x=x^{2} * x \text { and } x^{2} * x^{2}=x * x^{2} .
$$

From this and using right quasi-cancellative, we get $x=x^{2}$. Hence,

$$
x * y=x=x^{2}=x^{2} * y^{2}=x * y^{2}=x *(y * y)=y *(x * y)=y * x=y^{2} .
$$


That is,

$$
x=y * x \text {, and } y^{2}=x * y .
$$

It follows that $x=y$ from the right quasi-cancellative law. Therefore, $S$ is left quasi-cancellative.

Note that, in the proof of the first part of Theorem 4, the condition "AG**-groupoid" is not used, so we can get the following conclusion and the proof is omitted.

Theorem 5. Suppose that $(S, *)$ is an AG-groupoid. If $S$ is left quasi-cancellative, then it is right quasicancellative.

Definition 10. Assume that $(S, *)$ is an AG-groupoid. $S$ is called a power-cancellative AG-groupoid, if for any $a, b \in S, a^{2}=b^{2}$ implies $a=b$.

Obviously, every AG-band is power-cancellative. For the AG-groupoid $S$ in Example 2, it is power-cancellative, but it is not an AG-band. There exists some quasi-cancellative AG-groupoids which are not power-cancellative, such as Example 3. The following example shows that there exists some $\mathrm{AG}^{* *}$-groupoids which are not power-cancellative.

Example 4. Denote $S=\{1,2,3,4,5\}$, define operations $*$ on $S$ as Table 4 . We can verify that $(S, *)$ is a quasicancellative $A G^{* *}$-groupoid (see Example 1 in [9]), but it is not power-cancellative, since

$$
2 * 2=4 * 4, \text { but } 2 \neq 4 \text {. }
$$

Table 4. The operation * on non power-cancellative $S$.

\begin{tabular}{llllll}
\hline$*$ & $\mathbf{1}$ & $\mathbf{2}$ & $\mathbf{3}$ & $\mathbf{4}$ & $\mathbf{5}$ \\
\hline $\mathbf{1}$ & 1 & 1 & 1 & 1 & 1 \\
$\mathbf{2}$ & 1 & 2 & 2 & 4 & 5 \\
$\mathbf{3}$ & 1 & 2 & 3 & 4 & 5 \\
$\mathbf{4}$ & 1 & 5 & 5 & 2 & 4 \\
$\mathbf{5}$ & 1 & 4 & 4 & 5 & 2 \\
\hline
\end{tabular}

Definition 11. Suppose that $(S, *)$ is an AG-groupoid. $S$ is called a locally power-associative AG-groupoid, if for any $a \in S, a^{2} *\left(a^{2} * a^{2}\right)=\left(a^{2} * a^{2}\right) * a^{2}$.

Obviously, every AG-band is locally power-associative, every AG-3-band is locally powerassociative, every AG-4-band is locally power-associative. It is easy to verify that the AG-groupoid in Example 4 is locally power-associative. The following example shows that there exists some AG-groupoids which are not locally power-associative.

Example 5. Let $S=\{1,2,3,4\}$, define operations $*$ on $S$ as following Table 5 . We can verify that $(S, *)$ is an AG-groupoid, but it is not locally power-associative, since

$$
4^{2}=4 * 4=3, \text { but } 4^{2} *\left(4^{2} * 4^{2}\right)=4 \neq 3=\left(4^{2} * 4^{2}\right) * 4^{2} .
$$

Table 5 . The operation $*$ on non locally power-associative $S$.

\begin{tabular}{lllll}
\hline$*$ & $\mathbf{1}$ & $\mathbf{2}$ & $\mathbf{3}$ & $\mathbf{4}$ \\
\hline $\mathbf{1}$ & 2 & 1 & 3 & 4 \\
$\mathbf{2}$ & 3 & 4 & 2 & 1 \\
$\mathbf{3}$ & 4 & 3 & 1 & 2 \\
$\mathbf{4}$ & 1 & 2 & 4 & 3 \\
\hline
\end{tabular}


Theorem 6. Let $(S, *)$ be a power-cancellative and locally power-associative AG-groupoid. Then $S$ is left quasi-cancellative if and only if it is right quasi-cancellative.

Proof. By Theorem 5, if $S$ is left quasi-cancellative, then it is right quasi-cancellative.

Assume that $S$ is right quasi-cancellative, $x=x * y$ and $y^{2}=y * x, x, y \in S$.

By Lemma 1 (1), (2) and (3) we get that

$$
\begin{gathered}
x^{2}=x * x^{2}=\left(y^{2} * x^{2}\right) * x^{2}=\left(y^{2} * x^{2}\right) *(x * x)=\left(y^{2} * x\right) *\left(x^{2} * x\right)=x *\left(x^{2} * x\right) . \\
x^{2} * x^{2}=\left(x *\left(x^{2} * x\right)\right) * x^{2}=\left(x *\left(x^{2} * x\right)\right) *(x * x)=x^{2} *\left(\left(x^{2} * x\right) * x\right)=x^{2} *\left((x * x) * x^{2}\right)=x^{2} *\left(x^{2} * x^{2}\right) .
\end{gathered}
$$

By Definition $11, x^{2} *\left(x^{2} * x^{2}\right)=\left(x^{2} * x^{2}\right) * x^{2}$. It follows that

$$
x^{2} * x^{2}=x^{2} *\left(x^{2} * x^{2}\right) \text { and }\left(x^{2}\right)^{2}=x^{2} * x^{2}=\left(x^{2} * x^{2}\right) * x^{2} .
$$

From this and applying right quasi-cancellative, we get $x^{2} * x^{2}=x^{2}$. Hence,

$$
\left(x^{2}\right)^{2}=x^{2} * x^{2}=\left(x^{2} * x^{2}\right) * x^{2}=\left(x^{2} * x\right) *\left(x^{2} * x\right)=\left(x^{2} * x\right)^{2} .
$$

By Definition 10, we get that $x^{2}=x^{2} * x$. Moreover, using Lemma 1 again, we have

$$
\begin{gathered}
\left(y^{2} * x\right)^{2}=\left(y^{2} * x\right) * x=x^{2} * y^{2}=x^{2} ; \\
\left(x * y^{2}\right)^{2}=\left(x * y^{2}\right) *\left(x * y^{2}\right)=x^{2} *\left(y^{2} * y^{2}\right)=x^{2} * x=x^{2} .
\end{gathered}
$$

It follows that $\left(y^{2} * x\right)^{2}=\left(x * y^{2}\right)^{2}$. Using Definition 10, $\left(y^{2} * x\right)=\left(x * y^{2}\right)$. Hence,

$$
x=y^{2} * x(\text { Lemma } 1(3)) \text {, and } y^{2} * y^{2}=y^{2} * x=x * y^{2} .
$$

Applying the right quasi-cancellative law, we get $x=y^{2}$. Therefore,

$$
x=y^{2}=y * x \text {, and } y^{2} * y^{2}=x=x * y .
$$

Using the right quasi-cancellative law again, we have $x=y$. That is, $S$ is left quasi-cancellative.

\section{Conclusions}

In the paper, we studied quasi-cancellativity of AG-groupoids, and partially solved two conjectures. We proposed some new concepts of left(right) quasi-cancellative AG-groupoids, power-cancellative AG-groupoids, locally power-associative AG-groupoid and AG-(4,1)-bands. We investigated the relationships among them, and obtained some new results.

We completely solved Conjecture 2 in [9] and partially solved Conjecture 1 in [9], the main results are as following:

(1) Every AG-3-band is quasi-cancellative;

(2) Every AG-(4,1)-band is quasi-cancellative;

(3) For an $\mathrm{AG}^{*}$-groupoid, or an $\mathrm{AG}^{* *}$-groupoid, or a power-cancellative and locally power- associative AG-groupoid, it is left quasi-cancellative if and only if it is right quasi-cancellative;

(4) Every left quasi-cancellative AG-groupoid is right quasi-cancellative.

As the next research direction, we will study the combination structures of AG-groupoids (as non-associative semigroups), neutrosophic sets and related algebra systems (see [18-22]). 
Author Contributions: X.Z. proposed the idea of the research and wrote the draft, Y.M. and P.Y. completed the whole paper.

Funding: This research was funded by Scientific Research Program Funded by Shaanxi Provincial Education Department (No.18JK0099) and National Natural Science Foundation of China (No.61573240).

Conflicts of Interest: The authors declare no conflict of interest.

\section{References}

1. Kazim, M.A.; Naseeruddin, M. On almost semigroups. Alig. Bull. Math. 1972, 2, 1-7.

2. Holgate, P. Groupoids satisfying a simple invertive law. Math. Stud. 1992, 61, 101-106.

3. Mushtaq, Q. Zeroids and idempoids in AG-groupoids. Quasigroups Relat. Syst. 2004, 11, 79-84.

4. Protic, P.V.; Stevanovic, N. Abel-Grassmann's bands. Quasigroups Relat. Syst. 2004, 11, 95-101.

5. Mushtaq, Q.; Khan, M. Ideals in AG-band and AG*-groupoid. Quasigroups Relat. Syst. 2006, 14, $207-215$.

6. Shah, M.; Ali, A. Some structural properties of AG-group. Int. Math. Forum 2011, 6, 1661-1667.

7. Shah, M.; Shah, T.; Ali, A. On the cancellativity of AG-groupoids. Int. Math. Forum 2011, 6, 2187-2194.

8. Stevanovic, N.; Proti, P.V. Roots of AG-bands. In Approximation and Computation: In Honor of Gradimir V. Milovanovic, Springer Optimization and Its Applications 42; Gautschi, W., Ed.; Springer: New York, NY, USA, 2011; pp. 439-445.

9. Shah, M.; Ahmad, I.; Ali, A. On quasi-cancellativity of AG-groupoids. Int. J. Contemp. Math. Sci. 2012, 42, 2065-2070.

10. Khan, M.; Anis, S. On semilattice decomposition of an Abel-Grassmann's groupoid. Acta Math. Sin. Engl. Ser. 2012, 28, 1461-1468. [CrossRef]

11. Protic, P.V. Some remarks on Abel-Grassmann's groups. Quasigroups Relat. Syst. 2012, 20, 267-274.

12. Stevanovic, N.; Protic, P.V. Composition of Abel-Grassmann's 3-bands. Novi Sad J. Math. 2004, 34, $175-182$.

13. Dudek, W.A.; Gigon, R.S. Completely inverse AG**-groupoids. Semigroup Forum 2013, 87, 201-229. [CrossRef]

14. Khan, M.; Anis, S. An analogy of Clifford decomposition theorem for Abel-Grassmann groupoids. Algebra Colloq. 2014, 21, 347-353. [CrossRef]

15. Khan, M.; Smarandache, F.; Anis, S. Theory of Abel Grassmann's Groupoids; Educational Publisher: Columbus, OH, USA, 2015.

16. Rehman, N.; Park, C.; Ali Shah, S.I.; Ali, A. On generalized roughness in LA-semigroups. Mathematics 2018, 6, 112. [CrossRef]

17. Karaaslan, F. Some properties of $\mathrm{AG}^{*}$-groupoids and AG-bands under SI-product operation. J. Intell. Fuzzy Syst. 2018. [CrossRef]

18. Wu, X.Y.; Zhang, X.H. The decomposition theorems of AG-neutrosophic extended triplet loops and strong AG-(l,l)-loops. Mathematics 2019, 7, 268. [CrossRef]

19. Ma, Y.C.; Zhang, X.H.; Yang, X.F.; Zhou, X. Generalized neutrosophic extended triplet group. Symmetry 2019, 11, 327. [CrossRef]

20. Stanovský, D. Linear representation of Abel-Grassmann groups. Carpathian J. Math. 2017, 33, $257-263$.

21. Zhang, X.H.; Borzooei, R.A.; Jun, Y.B. Q-filters of quantum B-algebras and basic implication algebras. Symmetry 2018, 10, 573. [CrossRef]

22. Zhang, X.H.; Mao, X.Y.; Wu, Y.T.; Zhai, X.H. Neutrosophic filters in pseudo-BCI algebras. Int. J. Uncertain. Quan. 2018, 8, 511-526. [CrossRef]

(C) 2019 by the authors. Licensee MDPI, Basel, Switzerland. This article is an open access article distributed under the terms and conditions of the Creative Commons Attribution (CC BY) license (http://creativecommons.org/licenses/by/4.0/). 